\title{
An unusual case of isolate obstructive thrombi in the right atrium
}

\author{
Hassane Abdallah $^{1 *}$, François-Pierre Mongeon ${ }^{2}$ and Philippe Demers ${ }^{1}$ \\ ${ }^{1}$ Department of Cardiac Surgery, Montreal Heart Institute, Université de Montréal, Montreal, Quebec, Canada \\ ${ }^{2}$ Department of Cardiology, Montreal Heart Institute, Université de Montréal, Montreal, Quebec, Canada
}

\begin{abstract}
A 39-year-old female presented with recurrent dyspnea and was found on CT-scan to have a right atrial mass. Cardiac magnetic resonance accurately characterised the mass as a thrombi causing outflow obstruction. Surgical management was pursued with excision of the mass. It was unfortunately followed by a succession of thromboembolic event despite anticoagulation. The etiology of this rare cascade of events could not be accurately determined illustrating the difficulty in making a coagulation disorder diagnosis in an acute setting and with anticoagulation.
\end{abstract}

\section{Introduction}

Cardiac masses always represented a diagnostic challenge for clinicians. The distinction between the different types of intracardiac masses, including thrombi, vegetation and tumors, is an important step in providing therapeutic and prognostic information to the patient. Transthoracic 2D echocardiogram is the primary diagnostic tool used widely to detect and evaluate intracardiac mass and the cardiac MRI has become a useful tool to further characterize the mass. We are presenting a case in which the cardiac MRI was useful in the evaluation of an isolated mass obstructing tricuspid inflow in the right atrium.

\section{Case presentation}

A 39-year-old female was evaluated after initially consulting for complaint of dyspnea failing to improve prompting a CT study. The study demonstrated an atrial mass extending into the SVC without signs of pulmonary embolism. Her past medical history was limited to polycystic ovarian syndrome and two previous miscarriages. Her family history was positive for coronary heart disease, deep venous thrombosis and pulmonary embolism. Her thromboembolic risk factors included obesity, active smoking (35 pack-year history) and use of oral contraceptive pill.

Transthoracic echocardiogram (TTE) demonstrated a large immobile mass of $40 \times 25 \mathrm{~mm}$ attached to the right atrium, near the tricuspid valve with possible infiltration of the posterior leaflet. The mass was causing evidence of severe tricuspid stenosis by filling the tricusping inflow tract and thus creating a $15 \mathrm{mmHg}$ transvalvular gradient. Left Ventricular Ejection Fraction (LVEF) was maintained at $55 \%$. Transesophageal echocardiogram (TEE) confirmed the previous findings and demonstrated extension of the mass within the superior vena cava (Figure 1).

To further characterise the mass, a cardiac magnetic resonance (CMR) imaging study was completed. On T2*-weighted gradient echo imaging, there were areas of signal loss within the mass, suggesting the presence of hemosiderin. First-pass perfusion CMR showed no uptake of gadolinium in the mass, evidence of an avascular structure. All these findings were highly suggestive of a thrombotic process rather than a cardiac myxoma or angiosarcoma (Figure 2).

Surgical management was chosen and the right-atrial mass was excised (Figure 3 ). The mass was attached near the septal leaflet of the tricuspid valve but without involvement of the valve itself. Multiples attachments to the free wall were excised and the resection of the mass was completed including the part extending into the SVC. Due to severe regurgitation following the resection, a tricuspid annulus was implanted then a bioprosthesis (Carpentier-Edwards Perimount 29 $\mathrm{mm}$ ) was finally needed.

On pathology, the specimen was composed of a main irregularly shaped mass with multiple small fragments. The histologic examination revealed to be a thrombus of degenerated aspect, rich in fibrin and with absence of neoplastic process or significant inflammation. The pedicle of the specimen was composed of organized fibrocicatricial material with a portion of atrial wall.

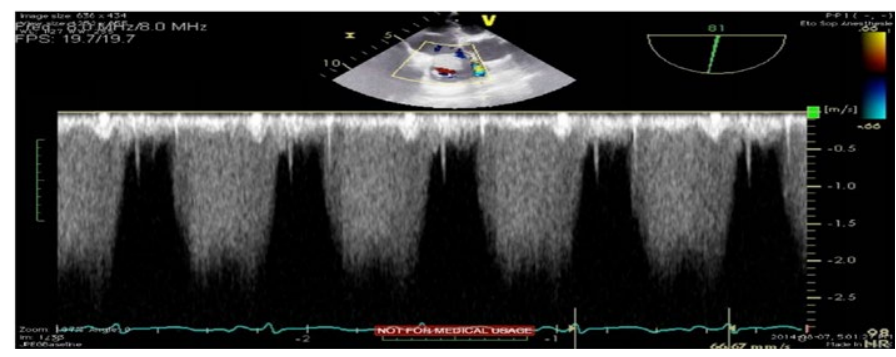

Figure 1. TEE demonstrating the presence of the mass within the right atrium.

Correspondence to: Hassane Abdallah, Montreal Heart Institute, 5000 Belanger Street, Montreal, Quebec H1T 1C8, Canada; Tel: 514 376-3330; Fax: 514 3761355; E-mail: abdallahhassane@hotmail.com

Key words: thrombosis, cardiac tumor, tricuspid valve, atrium

Received: July 10, 2015; Accepted: August 19, 2015; Published: August 24, 2015 

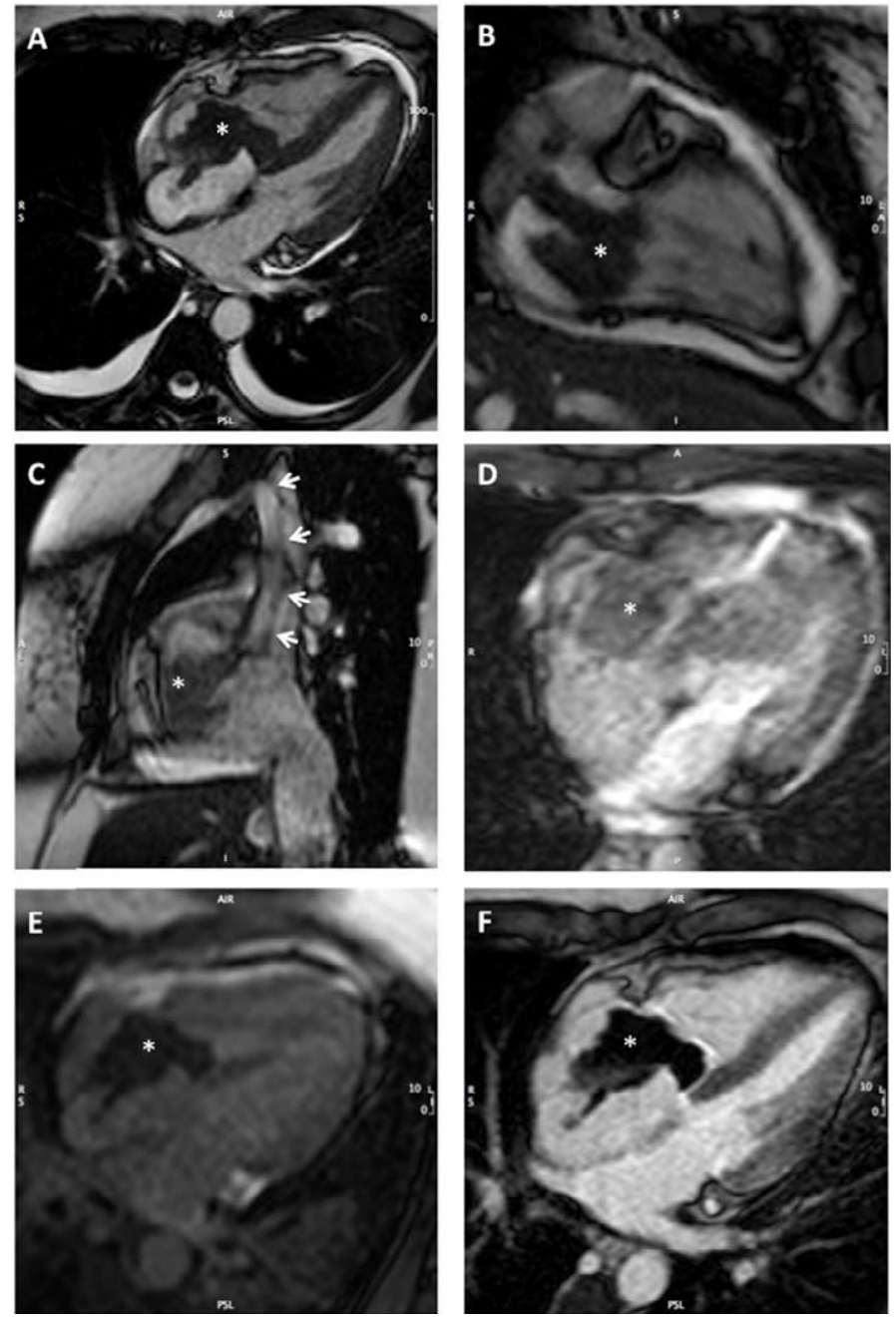

Figure 2. CMR imaging of the tricuspid valve mass (* in all panels). A) Cine CMR in the 4-chamber orientation. In addition to the large mass encasing the tricuspid valve, there is retraction of the free wall of the right atrium due to adherence of the mass. This systolic frame shows reversal of the interventricular septum curvature, indicating right ventricular pressure overload. There are also bilateral small pleural effusion and a mild non-compressive pericardial effusion, suggesting systemic venous hypertension. B) Cine $\mathrm{CMR}$ in the right ventricular 2-chamber orientation showing that the tricuspid valve mass in composed of 2 lobes. C) Cine CMR in the sagittal plane, showing the superior and inferior vena cava draining in the right atrium. The mass extends as a stalk (arrows) in the superior vena cava. D) $\mathrm{T} 2 *$-weighted gradient echo imaging shows areas of signal loss within the mass, consistent with the presence of hemosiderin and raising the hypothesis of a thrombotic mass. E) First-pass perfusion CMR shows no uptake of gadolinium in the mass (low signal), which is consistent with an avascular mass. F) The mass appears an area of signal void in late gadolinium enhancement imaging, which confirms the absence of delayed perfusion and the avascular nature of the mass. Collectively, these findings are consistent with a large thrombotic mass encasing the tricuspid valve.

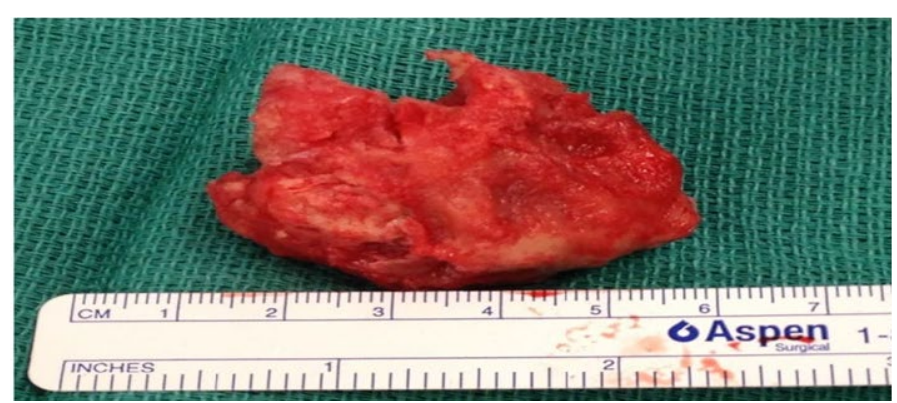

Figure 3. Intracardiac thrombus.
Post-surgery, the patient developed segmental peripheral pulmonary embolus despite therapeutic heparin. Therefore, heparin induced thrombocytopenia (HIT) was suspected and managed with change of anticoagulation to Argatroban. One week post-surgery, a thrombus on the bioprosthesic valve was discovered; for which she underwent three unsuccessful thrombolysis. Doppler ultrasonography revealed as well, bilateral brachial and radial thrombosis with necrotizing cutaneous zones, compatibles with subcutaneous arterial thrombosis.

On continued argatroban, a follow up TTE demonstrated resolution of the thrombus three weeks post-procedure in both the right atrium and SVC. Anticoagulation was changed to warfarin on discharge.

Initial investigation revealed a normal coagulation profile as well as normal serotonin, $\mathrm{CH} 100$ (complement activation), antithrombin III and S protein. Antiphospholipids antibodies (cardiolipins IgG and IgM), VDRL and Beta-HCG were negative. Measured post-operatively, activated $\mathrm{C}$ protein activity and antigen were diminished on two separate occasions (aCP: 0.47 and $0.64 \mathrm{~N}=0.70-1.20$; AgCP: 0.40 and $0.55 \mathrm{~N}=0.70-1.20)$. There was an absence of mutation concerning factor V Leiden, prothrombin 20210A and JAK-2V617F.

Antinuclear antibodies was positive at 1:160 dilution. Anti-DNA, anti-SM, anti-RNP, anti-SSA, anti-SSB antibodies and Rheumatoid Factor were negative.

\section{Discussion}

The presence of a right-atrial thrombus is a rare phenomenon that can potentially lead to devastating pulmonary embolism, hemodynamic instability and is associated with high mortality rate [1].

There exists a debate over the appropriate therapeutic approach. Options include thrombolytic therapy, surgical or percutaneous embolectomy and therapeutic anticoagulation [2]. In this case, it was decided to manage this patient surgically mainly because of the size of the mass and its effect on the tricuspid outflow.

This unusual thromboembolic event prompted investigations on the etiology of her suspected hypercoagulable state. The only remarkable result was a decrease in protein $\mathrm{C}$ activity and antigen. Protein $\mathrm{C}$ deficiencies is more prevalent as acquired than hereditary causes and has multiple etiology including active or recent thrombosis, surgical procedure, disseminated intravascular coagulation (DIC), anticoagulation on warfarin, severe sepsis and liver disease $[3,4]$. Having been measured only in the post-operative period, the $\mathrm{C}$ Protein level could easily represent an acquired deficiency from the inflammatory state post-surgery or secondary to the formation on the initial thrombus. Further testing of protein $\mathrm{C}$ level when the patient is no longer on his vitamin $\mathrm{K}$ antagonist (VKA) would be necessary to rule out a hereditary Protein $\mathrm{C}$ deficiency [3].

Behçet disease is reported as a possible cause of intracardiac thrombus presenting usually in the right atrium associated with pulmonary embolism and aneurysms. Although rare, intra-cardiac thrombosis indicates a worse prognosis within this disease [5]. Considering our patient had none of the other clinical finding of Behçet disease such as the oral ulceration or other organ involvements [6], it would be very surprising, in our opinion, that this intracardiac thrombus serves as initial presentation of this rare condition.

Antiphospholipid syndrome is a clinical entity loosely defined as the combination of a thromboembolic event and the presence of antiphospholipid antibodies (aPL) on laboratory investigation 
[7]. Deep venous thrombosis, arterial thrombosis and miscarriages are all clinical manifestations of the antiphospholipid syndrome which affected our patient over the years [7]. The presence of Lupus Anticoagulant (LA) could not be evaluated during the hospitalisation of our patient due to concomitant anticoagulation with unfractionated heparin then argobatran. Compared to aCL, which was negative in our patient, the LA assay has been reported to have the strongest association with clinical events in APS such as thrombosis. However, testing of our patient would be possible now under VKA with mixing studies using APTT and dRVVP for the presence of LA as suggested by the CLSI2014 and BCSH2012 guidelines [8]. This diagnosis remains the most likely etiology.

\section{Conclusion}

This case serves as an example of the difficulty in diagnosis of an intracardiac thrombus and the difficulty of investigating for the presence of a coagulation disorder associated in such an acute setting requiring immediate anticoagulation. Cardiac MRI has proven to be a high quality tool in evaluating the presence of such a mass within the heart cavities by accurately identifying it as a thrombus.

\section{References}

1. Rawat RS, Mehta Y, Arora D, Trehan N (2014) Asymptomatic type B right atrial thrombus in a case with protein S deficiency. Ann Card Anaesth 17: 237-239. [Crossref]

2. Yang JY, Williams S, Brandão LR, Chan AK (2010) Neonatal and childhood right atrial thrombosis: recognition and a risk-stratified treatment approach. Blood Coagul Fibrinolysis 21: 301-307. [Crossref]

3. Khor B, Van Cott EM (2010) Laboratory tests for protein C deficiency. Am J Hematol 85: 440-442. [Crossref]

4. Anderson JA, Weitz JI (2011) Hypercoagulable states. Crit Care Clin 27: 933-952, vii. [Crossref]

5. Gaffo AL (2013) Thrombosis in vasculitis. Best Pract Res Clin Rheumatol 27: 57-67. [Crossref]

6. Yurdakul S, Hamuryudan V, Yazici H (2004) Behçet syndrome. Curr Opin Rheumatol 16: 38-42. [Crossref]

7. Cervera R, Asherson RA (2003) Clinical and epidemiological aspects in the antiphospholipid syndrome. Immunobiology 207: 5-11. [Crossref]

8. Favaloro EJ, Wong RC (2014) Antiphospholipid antibody testing for the antiphospholipid syndrome: a comprehensive practical review including a synopsis of challenges and recent guidelines. Pathology 46: 481-495. [Crossref]

Copyright: (C2015 Abdallah H. This is an open-access article distributed under the terms of the Creative Commons Attribution License, which permits unrestricted use, distribution, and reproduction in any medium, provided the original author and source are credited. 